\title{
Transition probabilities of a string oscillator subject to impulsive collisions with a heavy mass point
}

\author{
Max Teubner ${ }^{\mathrm{a})}$ \\ Max-Planck-Institut für biophysikalische Chemie, Postfach 2841, 37077 Göttingen, Germany
}

(Received 1 June 2005; accepted 30 August 2005; published online 2 November 2005)

\begin{abstract}
Impulsive linear collisions between a string oscillator (a one-dimensional particle in a box) and a mass point are studied quantum mechanically. In the limit of a very heavy mass point (which corresponds classically to many collisions during a single encounter) the transition probabilities are determined exactly. The result permits a discussion of the mixed quantum-classical regime where the collider becomes almost classical while the oscillator remains quantum mechanical. While the average transition probabilities $P_{m \rightarrow n}$ are well reproduced by the Ehrenfest mean-field approximation, the prediction for the superimposed high-frequency resonance structure is qualitatively wrong for a genuine quantum oscillator. Only if the oscillator is also almost classical and if $(m-n)^{2} \sqrt{\mu} \ll m$, where $\mu$ is the mass ratio collider/oscillator, this structure is correctly predicted by the Ehrenfest approximation. (C) 2005 American Institute of Physics.
\end{abstract}

[DOI: $10.1063 / 1.2084946]$

\section{INTRODUCTION}

Vibrational-translational energy transfer is of central importance in understanding reaction dynamics. ${ }^{1,2}$ The problem remains theoretically challenging, since no simple exactly solvable model has been found which displays the crucial features of energy transfer. The simplest candidates (a harmonic oscillator which interacts with the colliding atom either by a hard sphere ${ }^{3}$ or an exponential interaction ${ }^{4}$ ) can be solved only numerically.

In the vibrational-translational energy transfer the dependence of the transition probabilities $P_{r s}$ on two parameters is of particular interest. The first is the oscillator frequency $\omega$ measured with respect to a suitable reference frequency $\omega / \omega_{0}$ and the second is the ratio

$$
\mu=\frac{m_{\mathrm{coll}}}{m_{\mathrm{osc}}}
$$

of collider mass to oscillator mass.

While the frequency is generally of great interest, the dependence on frequency becomes trivial for systems with hard-sphere interaction. The reason is that $\omega_{0}$ is something like the inverse duration of a collision, which is zero for hard-sphere interactions. For such systems the only nontrivial dependence is on the mass ratio $\mu$.

Small $\mu$ corresponds to light colliders which only weakly perturb the oscillator. This is the domain of Fermi's golden rule which is widely used by experimentalists. As $\mu$ increases, the interaction becomes stronger and its duration becomes longer and longer. For large mass ratios $\mu$ the interaction comprises multiple collisions and the theoretical treatment may become rather complicated.

From the numerical studies it is well known that the dependence of the $P_{r s}$ on $\mu$ is oscillatory, i.e., that there is a

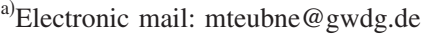

sequence of mass ratios $\mu_{n}$ where $P_{r s}$ vanishes (see Fig. 2). This is true classically as well as quantum mechanically. In the classical picture the quasiperiodic behavior is connected to the occurrence of multiple collisions during a single encounter.

In order to study the effect of a large mass ratio and (classically) multiple collisions on vibrational-translational energy transfer, we will choose a model with hard-sphere interaction.

The natural model here is the model of Shuler and Zwanzig $^{3}$ where a harmonic oscillator collides with a mass point. However, since the numerical procedure suggested by Shuler and Zwanzig runs into difficulties at large mass ratios, we will study an even simpler model.

The string oscillator (a one-dimensional particle in a box) is one of the very simplest quantum-mechanical objects. Many years ago Widom ${ }^{5,6}$ initiated the study of vibrationaltranslational energy transfer in this model by considering impulsive collisions with an impinging mass point.

While much is known about the string oscillator in the classical limit, ${ }^{7-9}$ the quantum model has only been studied rarely. ${ }^{10}$ It is the object of the present paper to show that this model can be solved in the limit of large collider mass. This opens the possibility of a comparison with the Ehrenfest mean-field approximation in the mixed quantum-classical regime.

\section{THE MODEL}

Consider a particle of mass $m_{\mathrm{osc}}$ in a box of length $L$ (the string oscillator). From the right a particle of mass $m_{\text {coll }}$ impinges on the oscillator. If $R$ and $r$ are the positions of collider and oscillator, respectively, the Hamiltonian reads 


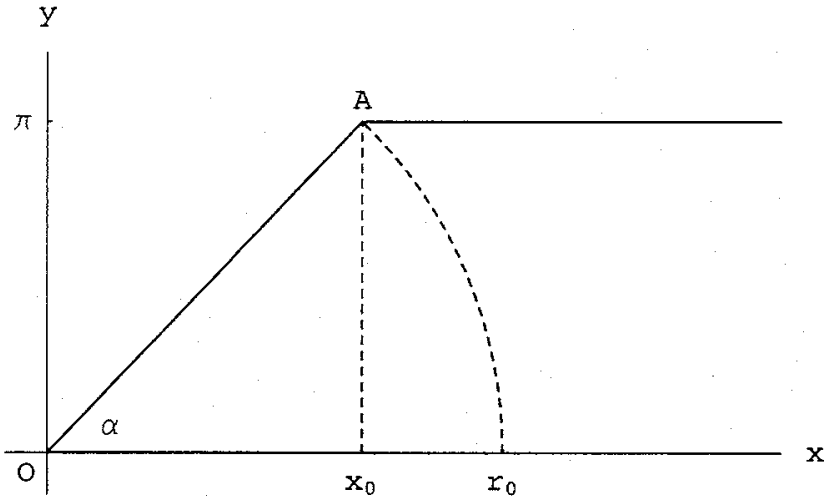

FIG. 1. Equation (5) must be satisfied in the enclosed region and must vanish on the boundary.

$$
H=-\frac{\hbar^{2}}{2 m_{\mathrm{coll}}} \frac{\partial^{2}}{\partial R^{2}}-\frac{\hbar^{2}}{2 m_{\mathrm{osc}}} \frac{\partial^{2}}{\partial r^{2}} .
$$

Because the oscillator is confined to the box, the wave function $\psi(R, r)$ must satisfy

$$
\psi(R, 0)=\psi(R, L)=0 .
$$

The additional boundary condition

$$
\psi(r, r)=0
$$

signifies the hard-core repulsion.

Similar to Hanson and Werbelow ${ }^{10}$ we rescale the coordinates by $x=\pi \sqrt{m_{\text {coll }} / m_{\text {osc }}} R / L$ and $y=\pi r / L$ and obtain the Helmholtz equation

$$
-\frac{\partial^{2}}{\partial x^{2}} \psi-\frac{\partial^{2}}{\partial y^{2}} \psi=\kappa^{2} \psi
$$

with the dimensionless wave number

$$
\kappa^{2}=\frac{2 m_{\mathrm{osc}} L^{2} E}{\pi^{2} \hbar^{2}}
$$

The Helmholtz equation [Eq. (5)] must be satisfied in the region $0<y<\pi, y<x \tan \alpha$, with

$$
\tan \alpha=\sqrt{\frac{m_{\text {osc }}}{m_{\text {coll }}}}=\mu^{-1 / 2},
$$

and must vanish on the boundary (see Fig. 1).

To the right of

$$
x_{0}=\frac{\pi}{\tan \alpha}
$$

the solution has the form

$$
\psi_{\mathrm{I}}(x, y)=e^{-i k_{m} x} \psi_{m}(y)+\sum_{n=0}^{\infty} R_{n} e^{i k_{n} x} \psi_{n}(y),
$$

where the $\psi_{n}(y)$ are the normalized eigenfunctions of the oscillator

$$
\psi_{n}(y)=\sqrt{\frac{2}{\pi}} \sin n y .
$$

Here $k_{m}$ is the wave vector of the incoming particle which impinges on an oscillator initially in state $m$. Energy conservation implies

$$
k_{n}^{2}+n^{2}=\kappa^{2} .
$$

Real $k_{n}$ represent outgoing waves which leave the oscillator in state $n$. The probability of a transition in which the oscillator changes from state $m$ to state $n$ is

$$
P_{m \rightarrow n}=\frac{k_{n}}{k_{m}}\left|R_{n}\right|^{2} .
$$

\section{METHOD OF SOLUTION}

In the circular segment $O r_{0} A$, where

$$
r_{0}=\frac{\pi}{\sin \alpha},
$$

the general solution in polar coordinates $r$ and $\varphi$ which satisfies the boundary conditions has the form ${ }^{10}$

$$
\psi_{\mathrm{II}}(r, \varphi)=\sum_{n=1}^{\infty} A_{n} J_{n \pi / \alpha}(\kappa r) \sin \frac{n \pi \varphi}{\alpha} .
$$

Solutions $\psi_{\mathrm{I}}$ and $\psi_{\mathrm{II}}$ overlap in the region $x_{0} r_{0} A$ where they must be identical. This may be implemented by demanding that $\psi_{\mathrm{I}}$ and $\psi_{\mathrm{II}}$ as well as their normal derivatives coincide on a path $\gamma$ which starts on the $x$ axis between $x_{0}$ and $r_{0}$ and ends at point $A$,

$$
\left.\psi_{\mathrm{I}}\right|_{\gamma}=\left.\psi_{\mathrm{II}}\right|_{\gamma},\left.\quad \frac{\partial \psi_{\mathrm{I}}}{\partial n}\right|_{\gamma}=\left.\frac{\partial \psi_{\mathrm{II}}}{\partial n}\right|_{\gamma} .
$$

For $\gamma$ Hanson and Werbelow ${ }^{10}$ use the line $x=x_{0}$ and postulate Eq. (15) on $2 n$ points on this line. This leads to a system on $2 n$ linear equations for the $R_{k}$ and $A_{k}$ which is solved numerically.

An alternative approach is to consider for $\gamma$ the circular segment $r_{0} A$. On this path we multiply Eq. (15) by $\sin (k \pi \varphi / \alpha)$ and integrate over $\varphi$ from 0 to $\alpha$. This leads to the linear system

$$
\begin{aligned}
& A_{k} J_{k \pi / \alpha}\left(\kappa r_{0}\right)=\sqrt{\frac{2}{\pi}}\left(\sum_{n} S_{k n} R_{n}+f_{k}\right), \\
& A_{k} \kappa J_{k \pi / \alpha}^{\prime}\left(\kappa r_{0}\right)=\sqrt{\frac{2}{\pi}}\left(\sum_{n} T_{k n} R_{n}+g_{k}\right),
\end{aligned}
$$

with matrices

$$
S_{k n}=2 \int_{0}^{1} e^{i k_{n} r_{0} \cos \alpha x} \sin \left(n r_{0} \sin \alpha x\right) \sin (k \pi x) d x,
$$




$$
\begin{aligned}
T_{k n}= & 2 \int_{0}^{1} e^{i k_{n} r_{0} \cos \alpha x}\left(i k_{n} \cos (\alpha x) \sin \left(n r_{0} \sin \alpha x\right)\right. \\
& \left.+n \sin (\alpha x) \cos \left(n r_{0} \sin \alpha x\right)\right) \sin k \pi x d x
\end{aligned}
$$

and vectors

$$
\begin{aligned}
f_{k}= & 2 \int_{0}^{1} e^{-i k_{m} r_{0} \cos \alpha x} \sin \left(m r_{0} \sin \alpha x\right) \sin (k \pi x) d x, \\
g= & 2 \int_{0}^{1} e^{-i k_{m} r_{0} \cos \alpha x}\left(-i k_{m} \cos (\alpha x) \sin \left(m r_{0} \sin \alpha x\right)\right. \\
& \left.+m \sin (\alpha x) \cos \left(m r_{0} \sin \alpha x\right)\right) \sin k \pi x d x .
\end{aligned}
$$

Eliminating the $A_{k}$ we obtain

$$
(Q S-T) R=g-Q f,
$$

where $Q$ is the diagonal matrix with elements

$$
q_{k}=\kappa \frac{J_{k \pi / \alpha}^{\prime}\left(\kappa r_{0}\right)}{J_{k \pi / \alpha}\left(\kappa r_{0}\right)} .
$$

Truncating Eq. (22) at some finite level $n$ supplies an alternative procedure for the numerical solution. For the same matrix size the accuracy is much higher than in the method of Hanson and Werbelow. ${ }^{10}$ The drawback is, however, that the matrices $S$ and $T$ and vectors $f$ and $g$ must be calculated numerically.

\section{TRANSITION PROBABILITY FOR LARGE COLLIDER MASS}

If the mass of the collider is much larger than the oscillator mass, the angle $\alpha$ is very small, and Eq. (22) may be solved by expanding in $\alpha$.

One finds after a brief calculation

$$
\begin{aligned}
& e^{-i k_{n} r_{0}} S_{k n}=\delta_{k n}+\alpha S_{k n}^{1}+O\left(\alpha^{2}\right), \\
& e^{-i k_{n} r_{0}} T_{k n}=i k_{n} \delta_{k n}+\alpha T_{k n}^{1}+O\left(\alpha^{2}\right),
\end{aligned}
$$

where

$$
S_{k n}^{1}=-i \pi k_{n} \int_{0}^{1} x^{2} \sin (n \pi x) \sin (k \pi x) d x
$$

and

$$
T_{k n}^{1}=2 n \int_{0}^{1} x \cos (n \pi x) \sin (k \pi x) d x+i k_{n} S_{k n}^{1} .
$$

The $f_{k}$ and $g_{k}$ are given by

$$
\begin{aligned}
& e^{i k_{m} r_{0}} f_{k}=\delta_{k m}-\alpha S_{k m}^{1}+O\left(\alpha^{2}\right), \\
& e^{i k_{m} r_{0}} g_{k}=-i k_{m} \delta_{k m}+\alpha T_{k m}^{1}+O\left(\alpha^{2}\right),
\end{aligned}
$$

where $m$ is again the index of the initial state.

Finally, we need the $q_{n}$ for small $\alpha$. From Ref. 11 we obtain for small $\alpha$

$$
q_{n} \sim u_{n}+\alpha v_{n}+O\left(\alpha^{2}\right),
$$

with
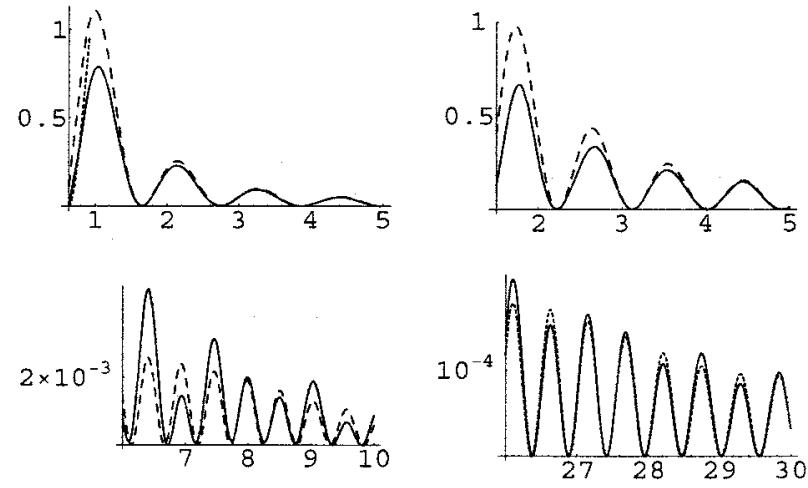

FIG. 2. Transition probabilities vs $\alpha^{-1}$. The full curves are numerically exact values and the broken curves correspond to Eq. (36). Upper left: $P_{1 \rightarrow 2}$ for $\kappa^{2}=6$. The dotted curve which ends at $\alpha^{-1}=0.9$ is Widom's approximation Ref. 6. Upper right: $P_{1 \rightarrow 2}$ for $\kappa^{2}=12$. Lower left and right: $P_{1 \rightarrow 3}$ for $\kappa^{2}$ $=12$.

$$
u_{n}=\left\{\begin{array}{cc}
i k_{n}, & \kappa<n \\
-k_{n} \tan \theta_{n}, & n<\kappa,
\end{array}\right.
$$

provided that $\kappa r_{0}$ stays away from the roots of $J_{n \pi / \alpha}(z) . \theta_{n}$ is given by

$$
\theta_{n}=\frac{\pi}{\alpha}\left(\sqrt{\kappa^{2}-n^{2}}-n \arccos \frac{n}{\kappa}\right)-\frac{\pi}{4} .
$$

We now insert $R_{k}=R_{k}^{0}+\alpha R_{k}^{1}$ into Eq. (22) and compare terms. At order 0

$$
R_{k}^{0}=-e^{-2 i k_{m} r_{0}} \frac{u_{m}+i k_{m}}{u_{m}-i k_{m}} \delta_{m k} .
$$

At this order there is only a phase shift without transitions.

At the next order one finds after some algebra for $n$ $\neq m$

$$
\begin{aligned}
R_{n}^{1}= & (-1)^{n+m} \frac{4 i}{\pi} e^{-i\left(k_{m}+k_{n}\right) r_{0}+i\left(\theta_{m}+\theta_{n}\right)} \\
& \times \frac{m n}{\left(m^{2}-n^{2}\right)^{2}} \frac{2 k_{m} k_{n} s_{m} s_{n}+\left(k_{m}^{2}+k_{n}^{2}\right) c_{m} c_{n}}{k_{n}} \alpha,
\end{aligned}
$$

with

$$
s_{n}=\sin \theta_{n}, \quad c_{n}=\cos \theta_{n} .
$$

This implies for the transition probabilities

$$
\begin{aligned}
P_{m \rightarrow n}= & \frac{1}{k_{n} k_{m}}\left(\frac { 4 } { \pi } \frac { m n } { ( m ^ { 2 } - n ^ { 2 } ) ^ { 2 } } \left(2 k_{m} k_{n} s_{m} s_{n}\right.\right. \\
& \left.\left.+\left(k_{m}^{2}+k_{n}^{2}\right) c_{m} c_{n}\right)\right)^{2} \alpha^{2}
\end{aligned}
$$

for $n \neq m$. In particular, $P_{m \rightarrow n}=P_{n \rightarrow m}$ as expected from detailed balance.

Figure 2 shows some transition probabilities $P_{n \rightarrow m}$ vs $\alpha^{-1}$. The full curves are numerically exact values and the broken curves correspond to Eq. (36). For moderate values of $\kappa$ the broken curves approach the true $P_{1 \rightarrow 2}$ even for quite large $\alpha$ (upper half of Fig. 2). For other indices Eq. (36) becomes applicable only for rather small $\alpha$ (lower half of Fig. 2). 


\section{COMPARISON WITH THE EHRENFEST APPROXIMATION}

Equation (36) supplies the exact transition probabilities in the limit of large collider mass. Systems where a part (typically an oscillator) is studied quantum mechanically, while the rest is assumed to behave classically, have found much interest recently (see, for example, Refs. 12 and 13).

The string model is an attractive toy to study the mixed quantum-classical dynamics on a very simple but nontrivial model. It permits an exact discussion of a model where the limit of large mass is performed on one, but not both of the particles. It differs from other similar models in the nonanalyticity of the interaction potential. As a consequence, the probability of nonadiabatic transitions decays only algebraically instead of exponentially. ${ }^{14,15}$

Consider the string model in the limit of large collider mass. Since the energy is fixed, the collider moves very slowly. The interaction with the quantum oscillator is only via the boundary conditions which also change very slowly. This suggests to compare the exact result of Eq. (36) with the Ehrenfest mean-field approach (see, for example, Tully ${ }^{12}$ ).

If the collider can be regarded as a classical particle, it interacts with the quantum oscillator only if its position $R(t)$ intrudes into the box $[0, L]$ where the oscillator lives. The oscillator then is subject to a moving wall during the time interval $0<t<T$ of intrusion where it satisfies

$$
i \hbar \frac{\partial \psi}{\partial t}=-\frac{\hbar^{2}}{2 m_{\mathrm{osc}}} \frac{\partial^{2} \psi}{\partial r^{2}},
$$

with the boundary conditions

$$
\psi(0, t)=\psi(R(t), t)=0, \quad 0<t<T .
$$

If initially the oscillator is in state $m$, adiabaticity indicates that it remains in this state during intrusion and therefore

$$
\psi(r, t)=\sqrt{\frac{2}{R(t)}} \sin \frac{m \pi r}{R(t)} e^{-i f(t)},
$$

with

$$
f(t)=\frac{\hbar}{2 m_{\mathrm{osc}}} m^{2} \pi^{2} \int_{0}^{t} R^{-2}\left(t^{\prime}\right) d t^{\prime} .
$$

The function $R(t)$ may be found from energy conservation

$$
\frac{m_{\mathrm{coll}}}{2} \dot{R}^{2}+\frac{\hbar^{2}}{2 m_{\mathrm{osc}}} \frac{m^{2} \pi^{2}}{R(t)^{2}}=E,
$$

where the second term is the energy eigenvalue of the adiabatic wave function. ${ }^{16}$ We write the solution in the form

$$
R(t)=L z\left(\frac{\omega t}{\sqrt{\mu}}\right),
$$

where

$$
\omega=\frac{\pi \hbar}{m_{\mathrm{osc}} L^{2}}
$$

is the characteristic frequency of the undisturbed string oscillator and

$$
z(x)=\sqrt{1+\kappa^{2} x^{2}-2 k_{m} x} .
$$

In particular, this implies for the duration of the interaction

$$
\omega T=\frac{2 k_{m}}{\kappa^{2}} \sqrt{\mu} .
$$

To determine the transition probabilities we insert the ansatz

$$
\psi=\sum_{n} \sqrt{\frac{2}{L}} a_{n}(t) \sin \frac{n \pi r}{R(t)}
$$

into Eq. (37). This leads to

$$
\dot{a}_{k}+i \frac{\hbar}{2 m_{\mathrm{osc}}} \frac{k^{2} \pi^{2}}{R(t)^{2}} a_{k}=\sum_{n} 2 \pi n c_{k n} a_{n} \frac{\dot{R}(t)}{R(t)},
$$

with

$$
c_{k n}=\int_{0}^{1} x \cos (n \pi x) \sin (k \pi x) d x .
$$

Denoting $a_{k}(t)$ by $a_{k}(\tau)$, with $\tau=\omega t / \sqrt{\mu}$, and integrating we obtain

$$
\begin{aligned}
& a_{k}(\tau) e^{(i \pi / 2) k^{2} \sqrt{\mu} w(\tau)}-a_{k}(0) \\
& =\sum_{n} 2 \pi n c_{k n} \int_{0}^{\tau} \frac{z^{\prime}\left(\tau^{\prime}\right)}{z\left(\tau^{\prime}\right)} a_{n}\left(\tau^{\prime}\right) e^{(i \pi / 2) k^{2} \sqrt{\mu} w\left(\tau^{\prime}\right)} d \tau^{\prime},
\end{aligned}
$$

where

$$
w(\tau)=\int_{0}^{\tau} \frac{d \tau^{\prime}}{z^{2}\left(\tau^{\prime}\right)} .
$$

On the right side we employ the assumption of adiabaticity. Then only the term with $n=m$ survives and for $k \neq m$,

$$
\begin{aligned}
& a_{k}(\tau) e^{(i \pi / 2) k^{2} \sqrt{\mu} w(\tau)} \\
& \quad=2 \pi m c_{k m} \int_{0}^{\tau} \frac{z^{\prime}\left(\tau^{\prime}\right)}{z\left(\tau^{\prime}\right)^{3 / 2}} e^{i \pi / 2\left(k^{2}-m^{2}\right) \sqrt{\mu} w\left(\tau^{\prime}\right)} d \tau^{\prime} .
\end{aligned}
$$

The Ehrenfest prediction for the transition probability $P_{m \rightarrow k}^{(E)}$ is $\left|a_{k}\left(\tau^{*}\right)\right|^{2}$, where $\tau^{*}=2 k_{m} / \kappa^{2}$. It becomes for large $\mu$

$$
P_{m \rightarrow n}^{(E)}=\left(\frac{8}{\pi} \frac{m n}{\left(m^{2}-n^{2}\right)^{2}} k_{m} \cos \xi_{m}\right)^{2} \mu^{-1},
$$

with

$$
\xi_{m}=\frac{\pi}{2}\left(n^{2}-m^{2}\right) \sqrt{\mu} w\left(\tau^{*}\right)=\frac{\pi}{2 m}\left(n^{2}-m^{2}\right) \sqrt{\mu} \arctan \frac{k_{m}}{m} .
$$

The Ehrenfest prediction has the correct $\mu$ dependence (recall that $\alpha^{2} \sim \mu^{-1}$ for large $\mu$ ) but differs from the exact 
result of Eq. (36) in the prefactor. In particular, detailed balance is not satisfied. Deviations between numerically exact quantum calculations and the Ehrenfest prediction have also been found for other oscillators. ${ }^{17-19}$

Are there any conditions where the Ehrenfest approximation to the transition probabilities becomes exact? Apparently it is not sufficient to let the mass of the collider tend to infinity.

It is well known that large mass is not sufficient for a particle to be "classical." From the WKB approximation (or the uncertainty relation $\Delta x \Delta p \sim \hbar$ ) it is obvious that no particle is classical near turning points. It is also known ${ }^{19}$ that the position uncertainty of the classical particle must be small if the Ehrenfest approximation is to be valid.

In the present case the kinetic energy of the collider should be large versus the energy of the oscillator. This implies $k_{n} \sim \kappa$ for all $n$. Replacing the $k_{n}$ by $\kappa$ one finds that Eqs. (36) and (52) become identical up to rapidly oscillating terms with frequency $\sim \sqrt{\mu}$ and zero mean. Even if the collider is almost classical, the Ehrenfest approximation predicts the transition probabilities only in some averaged sense. It fails to correctly predict the high-frequency resonance structure for a genuine quantum oscillator.

Are there any conditions (for a large but finite mass ratio $\mu$ ) where the Ehrenfest approximation is correct also for the high-frequency structure? This will be the case if ||$\theta_{n}-\theta_{m} \mid$ $-\xi_{m} \mid \ll 1$.

A brief calculation yields

$$
|| \theta_{n}-\theta_{m}\left|-\xi_{m}\right| \sim \frac{\pi^{2}}{4 m}(m-n)^{2} \sqrt{\mu},
$$

provided that $m, n \ll \kappa$. In particular, we must have

$$
m \gg \sqrt{\mu}
$$

and $m$ and $n$ must be large. The Ehrenfest high-frequency structure is correct only if the oscillator is almost classical and if

$$
|m-n|^{2} \sqrt{\mu} \ll m
$$

${ }^{1}$ J. Chesnoy and G. M. Gale, Adv. Chem. Phys. 70, 297 (1988).

${ }^{2}$ J. C. Owrutsky, D. Raftery, and R. M. Hochstrasser, Annu. Rev. Phys. Chem. 45, 519 (1994).

${ }^{3}$ K. E. Shuler and R. Zwanzig, J. Phys. Chem. 33, 1778 (1960).

${ }^{4}$ D. Secrest and B. R. Johnson, J. Chem. Phys. 45, 4556 (1966).

${ }^{5}$ B. Widom, J. Chem. Phys. 28, 918 (1955).

${ }^{6}$ B. Widom, J. Chem. Phys. 30, 238 (1959).

${ }^{7}$ M. P. Hanson and M. Blomme, J. Chem. Phys. 50, 4324 (1969).

${ }^{8}$ H. K. Shin, J. Chem. Phys. 62, 4130 (1975).

${ }^{9}$ D. Gutkowicz-Krusin, J. Chem. Phys. 67, 2830 (1977).

${ }^{10}$ M. P. Hanson and L. G. Werbelow, J. Chem. Phys. 58, 3669 (1973).

${ }^{11}$ I. S. Gradshteyn and I. M. Ryzhik, Table of Integrals, Series, and Products (Academic, New York, 1980).

${ }^{12}$ J. C. Tully, Faraday Discuss. 110, 407 (1998).

${ }^{13}$ K. Drucker, J. Comput. Phys. 153, 225 (1999).

${ }^{14}$ H. Nakamura, Nonadiabatic Transition (World Scientific, Singapore, 2002).

${ }^{15}$ M. Wilkinson and M. A. Morgan, Phys. Rev. A 61, 062104 (2000).

${ }^{16}$ Equation (41) can also be derived using the Hellman-Feynman force. Replacing the region behind the right wall by a constant potential $U$ $>E$, we have $\left\langle\psi\left|\nabla_{R} U\right| \psi\right\rangle=-U|\psi(R)|^{2}$. A short calculation shows that this becomes $-m_{\text {osc }}^{-1} \hbar^{2} \pi^{2} m^{2} R^{-3}$ in the limit of large $U$.

${ }^{17}$ P. Bala, P. Grochowski, B. Lesyng, and J. A. McCammon, in Quantum Mechanical Simulation Methods for Studying Biological Systems, Les Houches Workshop, 1996, edited by D. Bicout and M. Field (Springer Verlag, Berlin/Heidelberg, 1996).

${ }^{18}$ H. J. C. Berendsen and J. Mavri, in Quantum Mechanical Simulation Methods for Studying Biological Systems, Les Houches Workshop, 1996, edited by D. Bicout and M. Field (Springer Verlag, Berlin/Heidelberg, 1996).

${ }^{19}$ F. A. Bornemann, P. Nettesheim, and C. Schütte, J. Chem. Phys. 105, 1074 (1996) 